\title{
Impact of palladium nanoparticles (Pd-NPs) on the biology of neutrophils in vitro and on leukocyte attraction in vivo
}

\author{
Pierrette Kwemo • Abdelaziz Saafane • \\ Marion Vanharen • Isabelle Durocher • Denis Girard
}

Received: 18 June 2020 / Accepted: 4 November 2020 /Published online: 17 November 2020

(C) Springer Nature B.V. 2020

\begin{abstract}
Recently, palladium nanoparticles (Pd-NPs) have been shown to possess pro-inflammatory activities. Herein, we investigated potential in vitro effects of $\mathrm{Pd}-$ NPs (primary size of 1-10 nm) on the biology of neutrophils, key player cells in inflammation. Also, the aim of this study was to evaluate the pro-inflammatory activity of Pd-NPs using the murine air pouch model, a model previously proposed to be used as a standard assay for testing in vivo pro-inflammatory effects of NPs. Although the positive controls used in vitro give the expected results in all biological functions tested, Pd-NPs do not affect the production of reactive oxygen species and that of interleukin- $1 \beta$ (IL-1 $\beta$ ), IL-6, and IL8. Pd-NPs moderately increase cellular adhesion of neutrophils onto human endothelial cells and significantly increase the capacity of neutrophils to migrate and to delay apoptosis. We conclude that Pd-NPs possess some pro-inflammatory activity in vitro but do not attract leukocytes in vivo regardless of sex.
\end{abstract}

Keywords Palladium · Nanoparticles Inflammation · Neutrophils $\cdot$ Air pouch $\cdot$ Biomedicine

\footnotetext{
P. Kwemo · A. Saafane · M. Vanharen · I. Durocher ·

D. Girard $(\bowtie)$

Laboratoire de recherche en inflammation et physiologie des granulocytes, Université du Québec, Institut National de la Recherche Scientifique (INRS), Centre Armand-Frappier Santé Biotechnologie, 531 boul. des Prairies, Laval, QC H7V 1B7, Canada e-mail: denis.girard@inrs.ca
}

\section{Introduction}

Pd is best known for its use in the automobile industry as an active catalyst material in automobile catalytic converters. However, Pd is also used in other sectors, including electronics, jewelry, and dentistry, only to name a few (Ravindra et al. 2004). At the nanoscale, Pd-NPs possess interesting physicochemical properties, including thermal and chemical stability, electronic properties, photocatalytic activity, and optical properties (Phan et al. 2019). Pd-NPs are used in catalytic processes, electronics, wastewater treatment, and biomedicine. Of note, Pd-NPs are known to have great potential for the development of photothermal, photoacoustic, antitumor, and antimicrobial agents as well as gene and drug vectors, etc. However, there are increasing evidences that Pd-NPs are also able to induce undesired toxic effects in both in vitro and in vivo models (Leso and Iavicoli 2018; Phan et al. 2019). In vitro, Pd-NPs are known to exert cytotoxic and immunomodulatory effects. The studies focus mainly on the ability of Pd-NPs to induce apoptosis and the production of several cytokines, including pro-inflammatory cytokines (Boscolo et al. 2010; Reale et al. 2011). In vivo, some adverse effects of Pd-NPs have been reported on the renal, endocrine, and immune systems (Leso et al. 2018; Leso et al. 2019).

Inflammation is increasingly reported as one of the most undesired effect of nanoparticle (NP) exposures in both in vitro (Cho et al. 2012; Goncalves et al. 2010; Lu et al. 2009) and in vivo (Chen et al. 2013; Durocher and Girard 2016; Yuan et al. 2020) models. Although high 
neutrophil counts are frequently used as a marker of inflammation after NP exposure in several in vivo models, studies aiming at understanding the direct impact of NPs on the biology of neutrophils have been less investigated. To overcome this, in the last decade, we (Babin et al. 2013, 2015; Durocher et al. 2017; Goncalves and Girard 2014; Liz et al. 2015; Noel et al. 2016; Poirier et al. 2014, 2015) and others (Abrikossova et al. 2012; Dianzani et al. 2006; Moeller et al. 2012) have investigated how some NPs could alter the biology of human neutrophils, key player cells in inflammation and the most predominant leukocyte subtype found in the human blood. From these studies, it could be concluded that NPs possess the potential to alter the most important neutrophil responses involved in all steps of the inflammatory responses, including adherence, migration, phagocytosis, degranulation, reactive oxygen species (ROS) production (this is, however, not generalized), apoptosis, protein synthesis, and cytokine production. Although several NPs have been investigated by us and others, including titanium dioxide, cerium dioxide, zinc oxide, gadolinium oxide, and gold and silver NPs (Abrikossova et al. 2012; Babin et al. 2013; Durocher et al. 2017; Goncalves et al. 2010; Goncalves and Girard 2014) palladium NPs (Pd-NPs) have never been investigated in neutrophils. However, by studying different types of NPs, we recently documented that the capacity of eosinophils (the neutrophil sister cells) to adhere onto endothelial cells was increased after treatment with NPs, including Pd-NPs (Murphy-Marion and Girard 2018). Then, we demonstrated the importance of the actin cytoskeleton during Pd-NP-induced eosinophil adhesion as well as their inability to induce ROS production and apoptosis (Chhay et al. 2018). In this study, we reported how Pd-NPs can alter the biology of neutrophils and demonstrated their inability to attract neutrophils in vivo.

\section{Materials and methods}

\section{Chemicals}

RPMI-1640, HEPES, penicillin/streptomycin (P/S), $\mathrm{H}_{2} \mathrm{O}_{2}$, Viscum album agglutinin-I (VAA-I), lipopolysaccharides (LPS) from Escherichia coli, trypan blue, and dextran were purchased from Sigma-Aldrich Ltd. (Saint-Louis, Missouri); recombinant human tumor necrosis factor-alpha (TNF- $\alpha$ ) and interleukin-8 (IL-8) were purchased from PeproTech Inc. (Rocky Hill, NJ, USA).

\section{Pd-NPs}

The PD-NPs (product number 106PD) were purchased from Sciventions (Toronto, ONT) with a primary size of 1-10 nm, by transmission electronic microscopy, and were obtained at an original concentration of $1.5 \mathrm{mg} /$ $\mathrm{mL}$, stabilized by sodium polyacrylate. These Pd-NPs used in this study have been described previously and were used at a concentration of $100 \mu \mathrm{g} / \mathrm{mL}$, unless specified (Chhay et al. 2018). This concentration was selected according to our previous works showing that Pd-NPs induce human eosinophils adhesion, and in addition, this concentration was suitable not only for Pd-NPs but also with other NPs, allowing comparison within different NPs (Chhay et al. 2018; MurphyMarion and Girard 2018). In fact, several granulocyte functions can be altered at this concentration without activating cell necrosis in both human eosinophils and neutrophils. The endotoxin level of the Pd-NP suspension was determined by the classical Limulus amebocyte lysate (LAL) assay using the ToxinSensorTM Chromogenic LAL Endotoxin Assay Kit (Genscript Biotech Corp., Piscataway, NJ). Measurements were performed at a concentration of $100 \mu \mathrm{g} / \mathrm{mL}$. In addition, we incubated the Pd-NP suspension in Lysogeny broth agar plates for $72 \mathrm{~h}$ for testing sterility and observed the presence or absence of colonies. As a positive control, we used a suspension voluntary contaminated with human hear. Pictures of the plates were taken with a Motorola Moto $\mathrm{g}(7)$ play cell phone.

\section{Scanning electron microscopy}

For scanning electronic microscopy (SEM), a cover glass (disc) was cleaned with acetone and coated with $3 \mathrm{~nm}$ gold/palladium (Leica CPD300). Using a conductive silver paint (Ted Pella), the disc was fixed on a stub. A $100 \mu \mathrm{L}$ drop of the stock solution was put on the cover glass and let it dry. After drying, the sample was examined with a Hitachi Regulus 8220 scanning electron microscope.

Neutrophil isolation

Neutrophils were isolated from venous blood of healthy volunteers by dextran sedimentation, followed by 
centrifugation over Ficoll-Hypaque (Pharmacia Biotech, Inc., Quebec, Canada) as described previously (Babin et al. 2013). Blood donations were obtained from informed and consenting individuals according to institutionally approved procedures. In this study, blood was obtained from both men and women (age ranging from $\sim 20$ to 62 years old). Cell viability was monitored by trypan blue exclusion and found to be consistently $\geq$ $97 \%$. Cell purity ( $\geq 98 \%$ ) was verified by cytology from cytocentrifuged preparations colored by Hema-3 stain set (Biochemical sciences Inc., Swedesboro, NJ). Cell viability was evaluated systematically before and after each treatment. Neutrophils were then resuspended $10 \times 10^{6}$ cells $/ \mathrm{mL}$ in RPMI-HEPES $(25 \mathrm{mM})$, penicillin $(100 \mathrm{U} / \mathrm{mL}) /$ streptomycin $(100 \mu \mathrm{g} / \mathrm{mL})$ for all experiments, but ROS production (see under).

\section{Detection of intracellular ROS}

Cells $\left(10 \times 10^{6}\right.$ cells $\left./ \mathrm{mL}\right)$ were suspended in HBSS containing $10 \mu \mathrm{M} \mathrm{CM}-\mathrm{H}_{2}$ DCFDA (Invitrogen/Molecular Probes, Camarillo, CA) for $15 \mathrm{~min}$ at $37^{\circ} \mathrm{C}$ as published (Simard et al. 2011). Cells were then washed twice before being incubated in the presence of buffer or Pd-NPs for 5, 15, 30, and $60 \mathrm{~min}$. In these experiments, $\mathrm{H}_{2} \mathrm{O}_{2}$ (3\%, final concentration) was simply used as a positive technical control. ROS production was determined using a Spectra Max M5 plate reader (Molecular Devices, San Jose, CA) with an excitation/emission wavelength of $485 / 538 \mathrm{~nm}$ and was expressed as relative fluorescence units (RFU).

\section{Neutrophil cell adhesion assay}

The human umbilical vein cell line EA.hy926 (ATCC® CRL-2922 ${ }^{\mathrm{TM}}$ ) was purchased from American Type Culture Collection (Manassas, VA) and was grown in RPMI-1640 supplemented with $10 \%$ fetal calf serum and antibiotics. Cell viability was systematically evaluated before and after each treatment, and mortality never exceeded $5 \%$. These endothelial cells were used as a substratum for neutrophil cell adhesion. To do so, neutrophils were treated with buffer (Ctrl), Pd-NPs, or TNF- $\alpha$ (used as a positive control) (Murphy-Marion and Girard 2018; Pelletier and Girard 2005) for $30 \mathrm{~min}$ and then labeled for $30 \mathrm{~min}$ with $5 \mu \mathrm{M}$ calcein-AM (Molecular Probes, Inc., Eugene, OR, USA). The number of adherent neutrophils was calculated by counting the number of fluorescent cells from five randomly selected high-power fields $(\times 400)$ observed with a photomicroscope Leica DMRE equipped with an ebq $100 \mathrm{dc}$ epifluorescent condenser. Images were taken with a Cooke Sensicam High performance camera coupled to the Image Proplus ${ }^{\circledR}$ (version 4.0) program.

Cytokine production

The concentration of IL-8, IL- 6 , and IL-1 $\beta$ was determined using a commercially available ELISA kit for IL8, IL-6, or IL-1 $\beta$ (Life Technologies, Carlsbad, CA). Freshly isolated human neutrophils were incubated in the presence of buffer (Ctrl), Pd-NPs, or $1 \mu \mathrm{g} / \mathrm{mL}$ LPS (positive control) as above at $37^{\circ} \mathrm{C}$ in $5 \% \mathrm{CO}_{2}$ for $24 \mathrm{~h}$ in a 24-well plate containing RPMI-1640 supplemented with $5 \%$ heat-inactivated fetal calf serum. Supernatants were harvested after centrifugation and stored at $-70^{\circ} \mathrm{C}$ before performing ELISA.

\section{Assessment of neutrophil apoptosis}

Neutrophils $\left(10 \times 10^{6}\right.$ cells/mL in RPMI 1640-HEPES$\mathrm{P} / \mathrm{S}$, supplemented with $10 \%$ heat-inactivated autologous serum) were treated with buffer, Pd-NPs, or $1 \mu \mathrm{g} / \mathrm{mL}$ of the potent proapoptotic plant lectin Viscum album agglutinin-I (VAA-I) (Lavastre et al. 2002; Savoie et al. 2000) for $24 \mathrm{~h}$. Cytocentrifuged samples of neutrophils were prepared, stained with the Hema-3 Stain staining kit according to the manufacturer's instructions, and processed as documented previously (Lavastre et al. 2002).

Neutrophil migration

In vitro migration was performed in a 48-well microchamber (Neuro Probe Inc., Gaithersburg, MD, USA) using a 3-5 $\mu \mathrm{m}$ polycarbonate membrane filter as described previously (Durocher et al. 2017). The bottom wells were loaded (final volume, $25 \mu \mathrm{L}$ ) with buffer, Pd-NPs, or the positive control CXCL8/IL-8 $(25 \mathrm{nM})$, and the polycarbonate membrane was placed over the wells; then the top layer of the chamber was added over the membrane. Neutrophils $(50 \mu \mathrm{L}$ from a RPMI suspension of $1 \times 10^{6}$ cells $/ \mathrm{mL}$ ) were added into the upper wells. The chamber was incubated at $37^{\circ} \mathrm{C}$ for $60 \mathrm{~min}$ in a humidified incubator in the presence of $5 \%$ $\mathrm{CO}_{2}$. After the incubation, the top of the chamber was removed, and the upper side of the membrane was wiped carefully with the rubber scraper provided by 
the manufacturer. The polycarbonate membrane was fixed in methanol, colored with Hema 3 staining kit, mounted on a glass slide, and examined with a light microscope under $\times 400$ magnification. The number of cells in five random fields was counted, and the results were expressed as relative neutrophil migration (number of cells from tested group/number of cells from control).

Murine air pouch model

CD-1 male and female mice (6-8 weeks of age) were obtained from Charles River Laboratories (St-Constant, Canada). A period of acclimation of 1 week was allowed prior to initiation of experiments with the animals. On days 0 and 3, mice were anesthetized with isoflurane, and $3 \mathrm{~mL}$ of sterile air was injected subcutaneously, in the back, with a 26-gauge needle to form an air pouch as published previously (Durocher and Girard 2016; Durocher et al. 2017; Pelletier et al. 2004). On day 6, $1 \mathrm{~mL}$ of HBSS (Ctrl), 100 or $500 \mu \mathrm{g} / \mathrm{mL}$ of Pd-NPs, or $1 \mu \mathrm{g} / \mathrm{mL}$ LPS (positive control) was injected into the air pouch. Mice were killed by $\mathrm{CO}_{2}$ asphyxiation $6 \mathrm{~h}$ after the treatment, and the pouches were washed once with $1 \mathrm{~mL}$ and then twice with $2 \mathrm{~mL}$ of HBSS containing $10 \mathrm{mM}$ EDTA. Exudates were centrifuged at $100 \times \mathrm{g}$ for 0 min at $4{ }^{\circ} \mathrm{C}$, and cells were resuspended at $0.5 \times 10^{6}$ cells $/ \mathrm{mL}$, spread onto microscope slides, stained with Hema-Stain (Fisher Scientific, Ottawa, Canada) for identification of leukocyte cell subpopulations, and were counted with a hemocytometer. All experiments were performed as per protocols approved by Animal Use and Care Committees at INRS.

Statistical analyses

Statistical analyses were performed using repeated measures ANOVA, and differences between groups were assessed using the Dunnett's multiple comparison test with GraphPad Prism version 5.00 for Windows (GraphPad Software, San Diego, CA). Statistical significance was established at $p<0.05$.

\section{Results}

Sterility of Pd-NP suspension and ROS production

As assessed by the LAL assay (data not shown), the PdNP suspension reveals a level of endotoxins under the detection limit of $0.01 \mathrm{EU} / \mathrm{mL}(n=3)$. Since some NPs could interfere with classical LAL assay for determining endotoxin level (Dobrovolskaia and McNeil 2007), we incubated the Pd-NP suspension $(100 \mu \mathrm{g} / \mathrm{mL})$ in Lysogeny broth agar plates for $72 \mathrm{~h}$ for further confirming sterility. As illustrated in Fig. 1a, unlike the positive control, no colony was observed on the plate containing Pd-NP suspension. A SEM image of the Pd-NP suspension is illustrated in Fig. $1 \mathrm{~b}$ and indicates that most of the Pd-NPs possess a spherical form close to a size of $10 \mathrm{~nm}$. However, some aggregates (short and intermediate chain-like structures observable close to the white asterisk) are also observable agreeing with our previous results obtained by dynamic light scattering analysis (Chhay et al. 2018). Knowing the importance of ROS production by neutrophils (Dahlgren et al. 2019; Johansson et al. 1995), we next determined the impact of Pd-NPs on this biological response. Because Pd-NPs were previously found to increase or not ROS production in a variety of cells other than neutrophils, we decide to carefully determine this using 31 different blood donors (15 males +16 females). Figure 1 c shows that, unlike $\mathrm{H}_{2} \mathrm{O}_{2}$ that give the expected results, Pd-NPs do not increase ROS production after 5, 15, 30, and $60 \mathrm{~min}$ of treatment. To discriminate between a potential differential effect associated with sex, the data were separate taking this parameter into account. When comparing the results between neutrophils isolated from men and women (Fig. 1d), clearly, Pd-NPs do not induce a different response in male vs female neutrophils. In fact, the results were remarkably similar when plotted together (males and females) or separately (males or females alone). These results clearly indicate that Pd-NPs do not induce ROS production in human neutrophils. In addition, our experiments indicate that neutrophils isolated from men or women also respond in the same manner to $\mathrm{H}_{2} \mathrm{O}_{2}$.

Effect of Pd-NPs treatment on neutrophil adherence onto endothelial cells

As illustrated in Fig. 2a, Pd-NPs can slightly to moderately increase the capacity of neutrophils to adhere onto human endothelial cells. Although a clear trend is observed with a ratio of $1.5 \pm 0.6$ (mean $\pm \mathrm{SD}, n=11$, vs Ctrl), the results were not statistically significant. By comparison, the positive control TNF- $\alpha$ gives a ratio of $2.5 \pm 0.7$. 


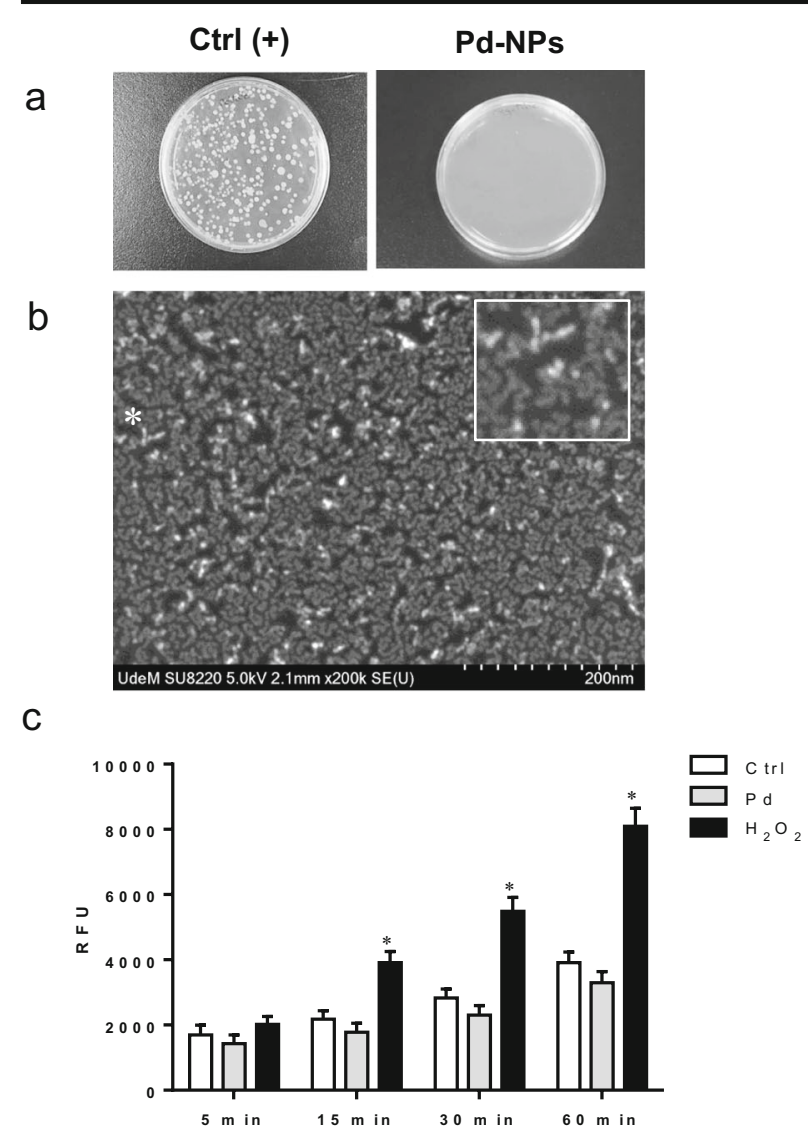

Fig. 1 Pd-NPs do not induce ROS production in human neutrophils. a Aliquots of a voluntary contaminated (human hair) or PdNP $(100 \mu \mathrm{g} / \mathrm{mL})$ suspension were deposited on Lysogeny broth agar plates and incubated for $72 \mathrm{~h}$. Sterility was confirmed by absence of any colony vs the positive control. Pictures of the plates were taken with a Motorola Moto $g(7)$ play cell phone and are representative of four experiments conducted at different periods of time during the whole study. b SEM image obtained as

Pd-NPs do not increase the production of IL-1 $\beta$, IL-6, and IL-8 in human neutrophils

As illustrated in Fig.3, Pd-NPs do not increase the production of IL-1 $\beta(n=23)$, IL-6 $(n=23)$, and IL-8 $(n=30)$ when compared with Ctrl. However, cells were responsive since LPS significantly increases the production of all the tested cytokines.

Modulation of neutrophil apoptosis and migration by Pd-NPs

As illustrated in Fig. 4a, Pd-NPs treatment lead to a weak to moderate, but significant, decrease of spontaneous neutrophil cell apoptosis from $50.2 \pm 15.6 \%$
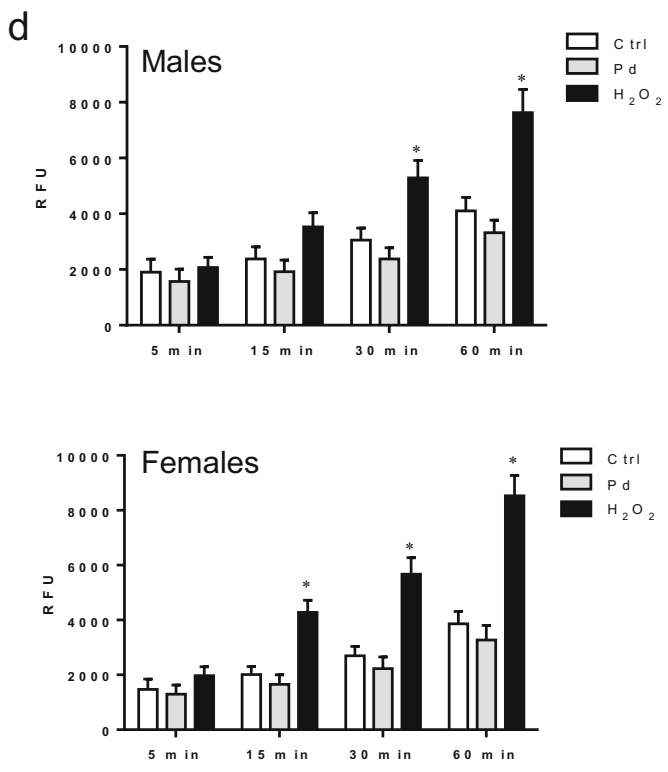

described in Materials and Methods. The region close to the white asterisk is zoomed out (top right part of the figure). $\mathbf{c}$ and $\mathbf{d}$ Freshly isolated human neutrophils were treated with buffer (Ctrl), Pd$\mathrm{NPs}$, or $\mathrm{H}_{2} \mathrm{O}_{2}$ for the indicated periods of time and ROS production, expressed as relative fluorescence units (RFU), and were determined as described in Materials and Methods. Results are expressed as means $\pm \mathrm{SD}$ (c, $n=31$ blood donors; $\mathbf{d}, n=15$ males or 16 females). $* p \leq 0.05$ vs Ctrl

(mean \pm SEM, $n=40$ ) to $40 \pm 14.3 \%$. As expected, VAA-I increases the apoptotic rate in almost $100 \%$ of cells. Figure $4 \mathrm{~b}$ shows that Pd-NPs increase the ability of neutrophils to migrate by a ratio of $1.6 \pm 0.5$ (mean \pm SEM, $n=22$, vs Ctrl). As expected, IL- 8 increases the migration by a ratio of $1.9 \pm 0.7$.

Pd-NPs do not induce acute inflammation in vivo in both male and female mice

Because a delay in neutrophil apoptosis and an increase capacity to migrate in vitro are two events known to be associated with inflammation, and since it is impossible to recreate inflammation in vitro, we next determined whether or not Pd-NPs could induce inflammation 
a

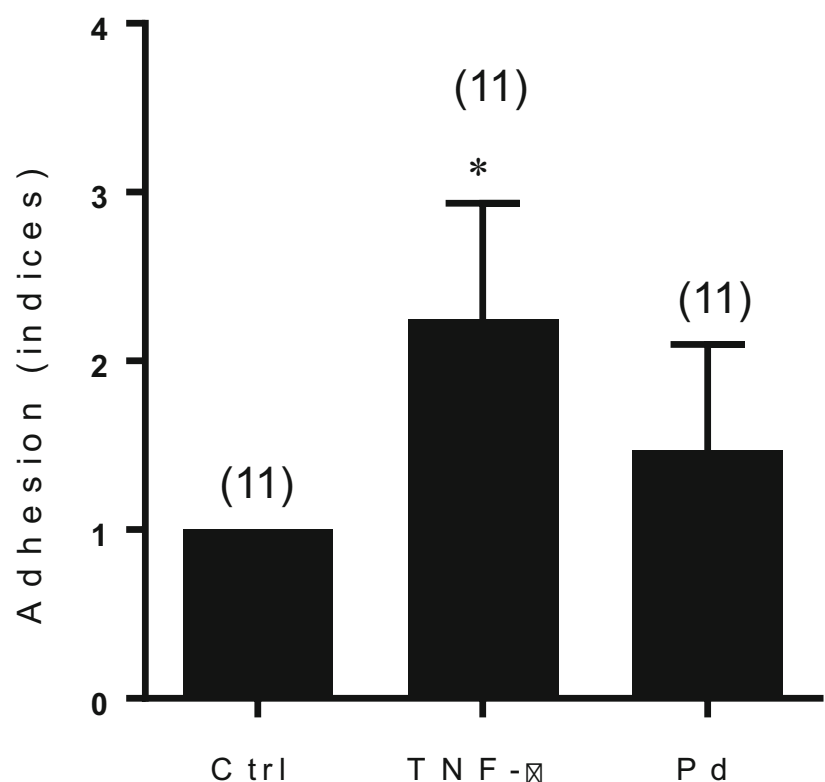

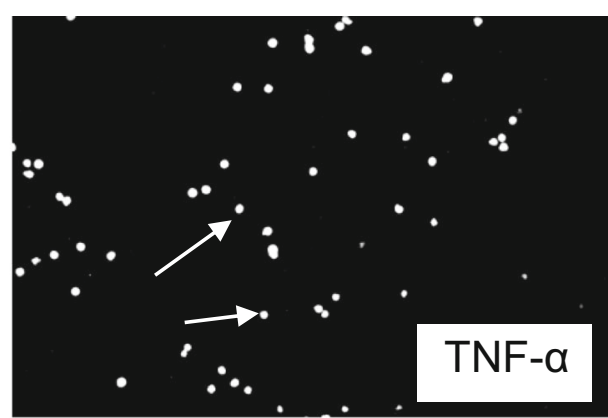

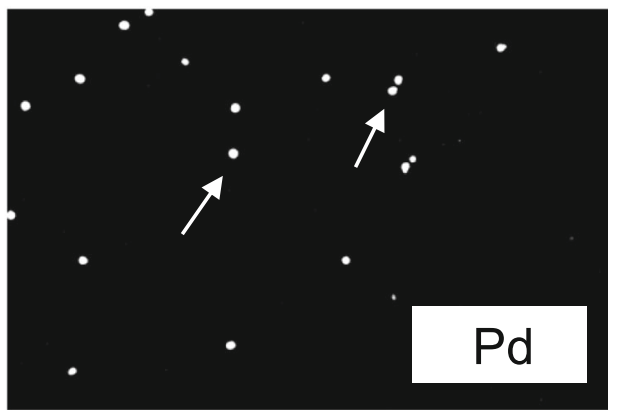

fluorescent neutrophil (white arrow) adhering onto endothelial cells. Results are mean $\pm \mathrm{SD}$, and the number of blood donors is indicated within parentheses. ${ }^{*} p \leq 0.05$ vs Ctrl set as 1

inflammation resulting by the infiltration of several millions of leukocytes into the exudates. Although no major difference was observed, LPS was slightly more efficient in female mice. By determining differential counts (Fig. 5b), PD-NPs, unlike LPS, do not preferentially attracted neutrophils, one of the two major leukocyte populations observed in this model, the other being mono-mac. a

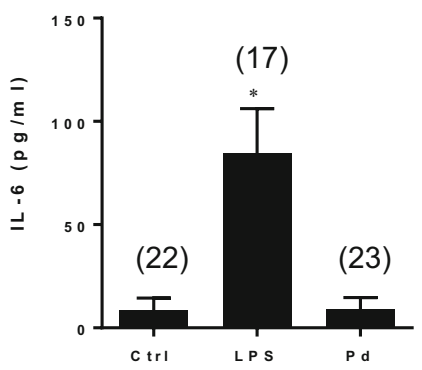

b

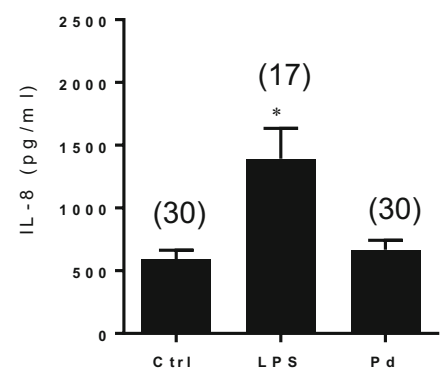

C

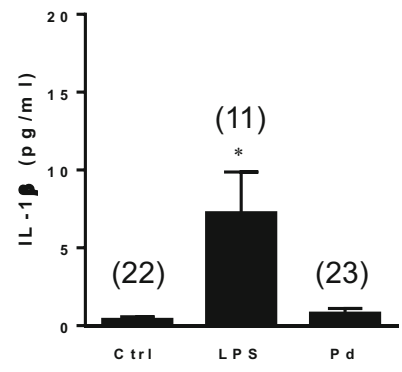

Fig. 3 Pd-NPs do not increase IL-8, IL-6, and IL-1 $\beta$ production in neutrophils. Freshly isolated human neutrophils were incubated for $24 \mathrm{~h}$ with buffer (Ctrl), LPS, or Pd-NPs, and the concentration of secreted cytokines was determined by ELISA as described in Materials and Methods. Results are mean $\pm \mathrm{SD}$, and the number of blood donors is indicated within parentheses. $* p \leq 0.05$ vs Ctrl 
a

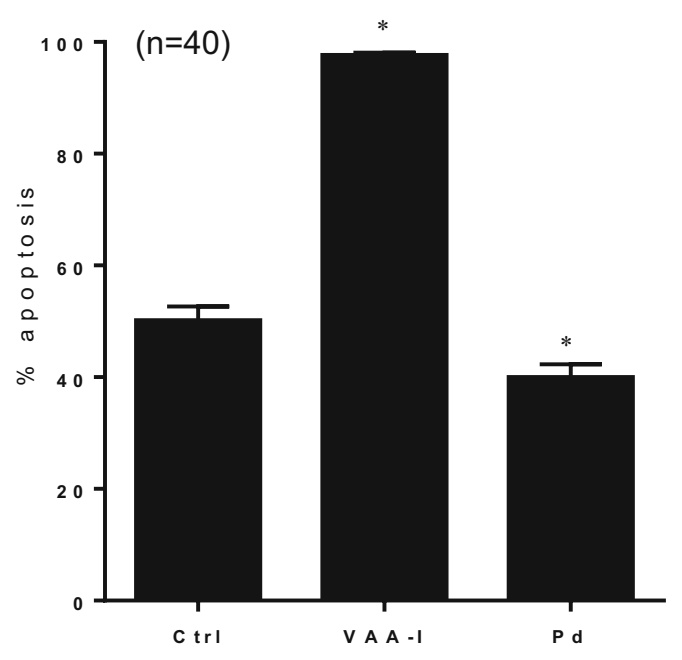

Fig. 4 Pd-NPs can delay human neutrophil spontaneous apoptosis and increase their capacity to migrate. Neutrophils were freshly isolated and incubated with the indicated agonists, and apoptosis (a) or migration (b) was determined after $24 \mathrm{~h}$ or $1 \mathrm{~h}$, respectively,

\section{Discussion}

A unique study reported pro-oxidative interactions of palladium metal with neutrophils; however, the metal source of Pd was not at the nanoscales (Theron et al. 2004). Therefore, the current study is the first one investigating the role of Pd-NPs on neutrophil cell biology and one of the very few investigating potential differences on neutrophil cell biology incorporating a sexbased analysis, all agents combined. Herein, the capacity of Pd-NPs to increase the adhesion of human neutrophils onto endothelial cells agrees with the previous data where Pd-NPs were found to increase the adhesion of human eosinophils onto a cell substratum (Chhay et al. 2018; Murphy-Marion and Girard 2018). However, in the present study, only a moderate (no significant) increase was observed after treatment with Pd-NPs. Of note, the same human umbilical vein cell line EA.hy926 was used in both studies, and yet, it is not clear why eosinophils (Chhay et al. 2018, Murphy-Marion and Girard 2018) adhere more efficiently onto these cells as compared to neutrophils (this report). It is highly plausible that differential expression and/or function of cell surface adhesion molecules is involved. In this respect, IL-4 was reported to induce adherence of human eosinophils and basophils but not neutrophils to b

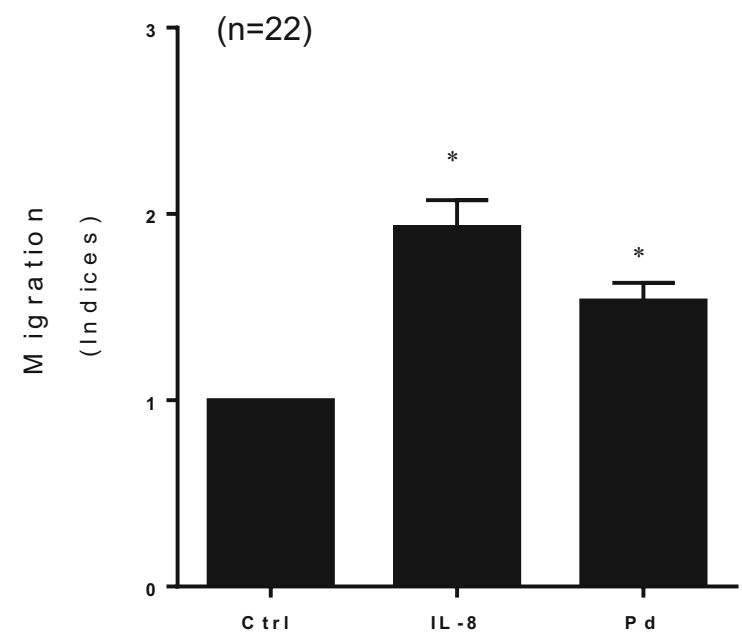

as described in Materials and Methods. Results are expressed as means $\pm \mathrm{SD}$ (a, $n=40$ blood donors; $\mathbf{b}, n=22$ blood donors $) * p \leq$ 0.05 vs Ctrl

endothelium, and this was associated with expression of VCAM-1 (vascular cell adhesion molecule-1) known to counteract with the integrin VLA-4 (very late antigen-4) not or very weakly expressed in human neutrophils (Schleimer et al. 1992). Interestingly, the capacity of Swiss mouse 3T3 fibroblasts to adhere onto gold, platinum, and palladium surfaces was previously reported to be differently modulated (Turner et al. 2004). Cells on palladium surfaces were more weakly attached as compared to gold and platinum. Whether or not adhesion onto these surfaces could also be a cell-specificdependent mechanism would be important to determine in the future.

We found that Pd-NPs do not induce the production of ROS in human neutrophils. These results agree with those observed in human eosinophils (Chhay et al. 2018) and other primary human cells such as bronchial epithelial cells (Wilkinson et al. 2011) and different human immortalized cells including alveolar carcinoma cell line A549 (Wilkinson et al. 2011), eosinophilic cell line AML-14.3D10 (Chhay et al. 2018), epithelial colorectal adenocarcinoma cell line Caco-2 (Hildebrand et al. 2010), and keratinocyte cell line HaCaT (Hildebrand et al. 2010). In contrast, others reported that Pd-NPs could increase ROS production in rat embryo fibroblast cell line Rat-1 and human lung carcinoma 


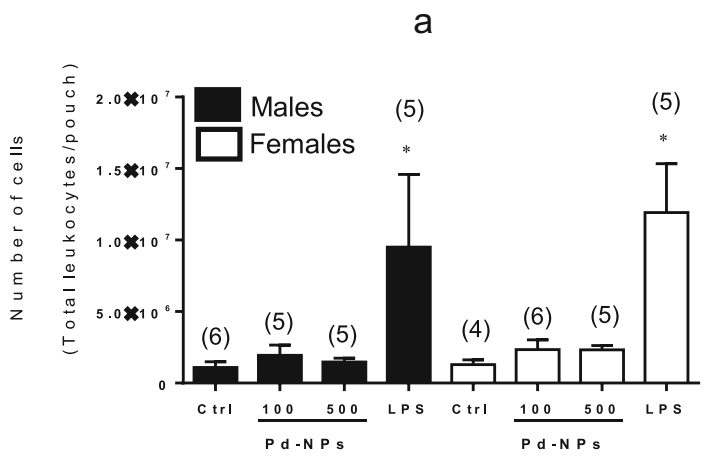

Fig. 5 Pd-NPs do not induce leukocyte infiltration in vivo. After creation of air pouches, buffer, LPS, or 100 and $500 \mu \mathrm{g} / \mathrm{mL}$ PdNPs were directly administered into murine air pouches, and exudates were harvested after $6 \mathrm{~h}$. The total leukocyte numbers (a) and differential counts (\% of monocytes-macrophages (mono-

epithelial cell line A549 (Iavicoli et al. 2017), human monocyte-like cell line THP-1 (Neubauer et al. 2015), human ovarian cancer cell line A2780 (Gurunathan et al. 2015), human skin malignant melanoma cell line A375 (Alarifi et al. 2017), and human peripheral blood mononuclear cells (Petrarca et al. 2014). However, in this latter study, the ROS production was only moderately and nonsignificantly increased. The experiments were performed with only three donors, and cells were incubated with Pd-NPs in vitro for $48 \mathrm{~h}$. Herein, we incubated cells with Pd-NPs for 0-60 min only since we were interested in the respiratory burst occurring via NADPH activation, the main source of ROS in neutrophils. In addition, we have performed 31 different experiments (31 different blood donors: 15 men and 16 women) to conclude inability of inducing ROS production regardless of sex. Further, our results are supported by the fact that Pd-NPs do not induce neutrophil apoptosis, a biological process frequently associated with an initial ROS production. Of note, in the above studies reporting that Pd-NPs induce ROS production in a b
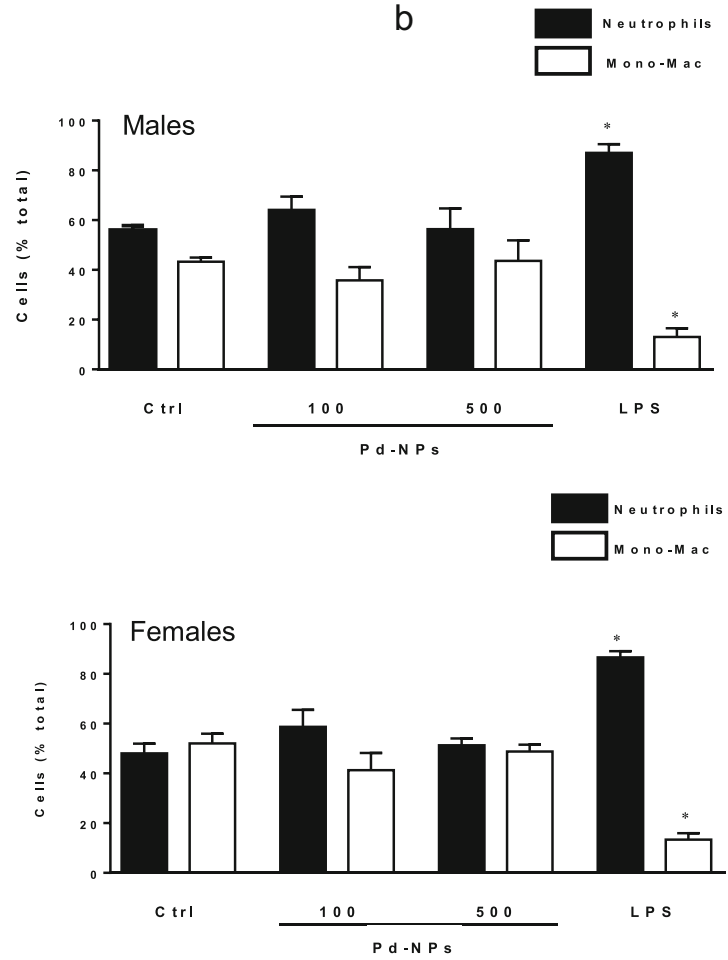

mac) and neutrophils in males and female (b) were then determined as described in Materials and Methods. Results are mean \pm $\mathrm{SD}$, and the number of mice is indicated within parentheses. $* p \leq$ 0.05 vs Ctrl

variety of cell types, cytotoxicity and/or apoptosis was also observed (Alarifi et al. 2017; Gurunathan et al. 2015; Hildebrand et al. 2010; Iavicoli et al. 2017). Herein, Pd-NPs rather delay neutrophil apoptosis and do not induce cell necrosis.

Pd-NPs were found to increase the ability of neutrophils to migrate. Although a protocol similar to the one used in the current study has been established by others (Skoczen et al. 2011), the literature is lacking regarding the ability of NPs do induce neutrophil migration/ chemotaxis in vitro. However, the present results agree with our previous study in which gold NPs were also found to increase migration of neutrophils (Durocher et al. 2017). Using the murine alveolar macrophage cell line $\mathrm{J} 774.2$, one study reported that carbon black NPs moderately increased macrophage migration in vitro using a system like the one used by us (Barlow et al. 2005). Of note, when carbon black NPs were incubated with serum, the response was markedly increased. In our hands, serum was not added. It is difficult to explain how Pd-NPs could attract neutrophils during the 
migration assay used. Indeed, in our system, as most other ones, neutrophils normally undergo morphological cell shape changes to pass through the filter. We cannot exclude the possibility that some neutrophils could nonspecifically pass via simple gravity (observable in the negative control) and that in presence of $\mathrm{Pd}-$ NPs would degranulate and/or produce some potent chemo-attractants. This remains to be determined, but previously, we demonstrated that some NPs could induce degranulation in human neutrophils (Babin et al. 2013). When testing the capacity to produce and secrete cytokines in Pd-NP-induced neutrophils, we did not observe increase levels of IL- $1 \beta$, IL- 6 , and IL- 8 when compared to controls. However, as expected, LPS increased cytokine production. This further supports the results obtained with the LAL assay and the agar plate indicating absence of contaminated endotoxins in PdNPs; otherwise, the production of these cytokines would have been increased (particularly IL-6 and IL-8). It is important to mention here that our results do not rule out the possibility that Pd-NPs could modulate the production of cytokines other than those tested in the current study. This remains to be determined.

The murine air pouch model of acute inflammation was recently proposed to be added as a future standard assay for testing in vivo pro-inflammatory effects of several types of NPs (Girard 2014; Vandooren et al. 2013). This model is especially useful to determine the pro-inflammatory activity of an agent when administered into the air pouch, giving general information on the inflammatory response including the three important phases, namely, initiation, amplification, and resolution depending on the dose-response and kinetics studied. We used this model for several years for testing different types of agents, including more recently NPs where some were found to be pro-inflammatory and others not, as judged by the leukocyte infiltration, especially neutrophils (Durocher and Girard 2016; Durocher et al. 2017; Goncalves and Girard 2011, 2013). However, we never incorporated a sex-based analysis. The current study indicates that Pd-NPs, in the experimental conditions used, do not induce leukocyte infiltration regardless of sex, but LPS induces a more pronounced effect in female mice when compared to males. This agrees with the general immune scenario in which, in general, innate and adaptive immune responses are more potent in females than males. Females are, however, less susceptible to many types of infection but in contrast are more prone to develop autoimmune diseases (Jaillon et al.
2019; Klein and Flanagan 2016). Although Pd-NPs did not induce a neutrophilic infiltration by themselves, it is possible that, as we have documented for fullerenols (polyhydroxylated $\mathrm{C}_{60}$ Fullerene NPs), Pd-NPs could act in concert with other agents, namely, LPS, for causing inflammation, a situation that is likely to occur in vivo during infections. In this respect, further studies need to be performed.

In conclusion, because Pd-NPs can alter the biology of human neutrophils, and knowing that differences observed between men and women immune responses and inflammation have been previously reported, we recommend to, whenever possible, systematically test in parallel in vitro and/or in vivo potential impact of a given NPs by incorporating sex-based analysis into future studies. This will certainly not only increase our knowledge on the mode of action of a given NP but will also improve our understanding of sex as an important variable that has to be considered in nanotoxicology or related fields.

Funding The study was supported by grants from the Institut de recherche Robert-Sauvé en santé et en sécurité du travail (IRSST).

\section{Compliance with ethical standards}

Conflict of interest The authors declare that they have no conflict of interest.

\section{References}

Abrikossova N, Skoglund C, Ahren M, Bengtsson T, Uvdal K (2012) Effects of gadolinium oxide nanoparticles on the oxidative burst from human neutrophil granulocytes. Nanotechnology 23:0957

Alarifi S, Ali D, Alkahtani S, Almeer RS (2017) ROS-mediated apoptosis and genotoxicity induced by palladium nanoparticles in human skin malignant melanoma cells. Oxidative Med Cell Longev 2017:8439098

Babin K, Antoine F, Goncalves DM, Girard D (2013) TiO2, CeO2 and $\mathrm{ZnO}$ nanoparticles and modulation of the degranulation process in human neutrophils. Toxicol Lett 221:57-63

Babin K, Goncalves DM, Girard D (2015) Nanoparticles enhance the ability of human neutrophils to exert phagocytosis by a Syk-dependent mechanism. Biochim Biophys Acta 1850: 2276-2282

Barlow PG, Donaldson K, MacCallum J, Clouter A, Stone V (2005) Serum exposed to nanoparticle carbon black displays increased potential to induce macrophage migration. Toxicol Lett 155:397-401 
Boscolo P, Bellante V, Leopold K, Maier M, Di Giampaolo L, Antonucci A, Iavicoli I, Tobia L, Paoletti A, Montalti M, Petrarca C, Qiao N, Sabbioni E, Di Gioacchino M (2010) Effects of palladium nanoparticles on the cytokine release from peripheral blood mononuclear cells of non-atopic women. J Biol Regul Homeost Agents 24:207

Chen H, Dorrigan A, Saad S, Hare DJ, Cortie MB, Valenzuela SM (2013) In vivo study of spherical gold nanoparticles: inflammatory effects and distribution in mice. PLoS One 8:e58208

Chhay P, Murphy-Marion M, Samson Y, Girard D (2018) Activation of human eosinophils with palladium nanoparticles (Pd NPs): importance of the actin cytoskeleton in Pd NPs-induced cellular adhesion. Environ Toxicol Pharmacol 57:95-103

Cho WS, Duffin R, Poland CA, Duschl A, Oostingh GJ, Macnee W, Bradley M, Megson IL, Donaldson K (2012) Differential pro-inflammatory effects of metal oxide nanoparticles and their soluble ions in vitro and in vivo; zinc and copper nanoparticles, but not their ions, recruit eosinophils to the lungs. Nanotoxicology 6:22-35

Dahlgren C, Karlsson A, Bylund J (2019) Intracellular neutrophil oxidants: from laboratory curiosity to clinical reality. J Immunol 202:3127-3134

Dianzani C, Cavalli R, Zara GP, Gallicchio M, Lombardi G, Gasco MR, Panzanelli P, Fantozzi R (2006) Cholesteryl butyrate solid lipid nanoparticles inhibit adhesion of human neutrophils to endothelial cells. Br J Pharmacol 148:648-656

Dobrovolskaia MA, McNeil SE (2007) Immunological properties of engineered nanomaterials. Nat Nanotechnol 2:469-478

Durocher I, Girard D (2016) In vivo proinflammatory activity of generations 0-3 (G0-G3) polyamidoamine (PAMAM) nanoparticles. Inflamm Res 65:745-755

Durocher I, Noel C, Lavastre V, Girard D (2017) Evaluation of the in vitro and in vivo proinflammatory activities of gold $(+)$ and gold (-) nanoparticles. Inflamm Res 66:981-992

Girard D (2014) Using the air pouch model for assessing in vivo inflammatory activity of nanoparticles. Int J Nanomedicine 9: 1105

Goncalves DM, Girard D (2011) Titanium dioxide (TiO(2)) nanoparticles induce neutrophil influx and local production of several pro-inflammatory mediators in vivo. Int Immunopharmacol 21:21

Goncalves DM, Girard D (2013) Evidence that polyhydroxylated C60 fullerenes (fullerenols) amplify the effect of lipopolysaccharides to induce rapid leukocyte infiltration in vivo. Chem Res Toxicol 26:1884-1892

Goncalves DM, Girard D (2014) Zinc oxide nanoparticles delay human neutrophil apoptosis by a de novo protein synthesisdependent and reactive oxygen species-independent mechanism. Toxicol in Vitro 28:926-931

Goncalves DM, Chiasson S, Girard D (2010) Activation of human neutrophils by titanium dioxide ( $\mathrm{TiO} 2)$ nanoparticles. Toxicol in Vitro 24:1002-1008

Gurunathan S, Kim E, Han JW, Park JH, Kim JH (2015) Green chemistry approach for synthesis of effective anticancer palladium nanoparticles. Molecules 20:22476-22498

Hildebrand H, Kuhnel D, Potthoff A, Mackenzie K, Springer A, Schirmer K (2010) Evaluating the cytotoxicity of palladium/ magnetite nano-catalysts intended for wastewater treatment. Environ Pollut 158:65-73
Iavicoli I, Farina M, Fontana L, Lucchetti D, Leso V, Fanali C, Cufino V, Boninsegna A, Leopold K, Schindl R, Brucker D, Sgambato A (2017) In vitro evaluation of the potential toxic effects of palladium nanoparticles on fibroblasts and lung epithelial cells. Toxicol in Vitro 42:191-199

Jaillon S, Berthenet K, Garlanda C (2019) Sexual dimorphism in innate immunity. Clin Rev Allergy Immunol 56:308-321

Johansson A, Jesaitis AJ, Lundqvist H, Magnusson KE, Sjolin C, Karlsson A, Dahlgren C (1995) Different subcellular localization of cytochrome $\mathrm{b}$ and the dormant NADPH-oxidase in neutrophils and macrophages: effect on the production of reactive oxygen species during phagocytosis. Cell Immunol $161: 61-71$

Klein SL, Flanagan KL (2016) Sex differences in immune responses. Nat Rev Immunol 16:626-638

Lavastre V, Pelletier M, Saller R, Hostanska K, Girard D (2002) Mechanisms involved in spontaneous and Viscum album agglutinin-I-induced human neutrophil apoptosis: Viscum album agglutinin-I accelerates the loss of antiapoptotic Mcl1 expression and the degradation of cytoskeletal paxillin and vimentin proteins via caspases. J Immunol 168:1419

Leso V, Iavicoli I (2018) Palladium nanoparticles: toxicological effects and potential implications for occupational risk assessment. Int J Mol Sci 19(2):503. https://doi.org/10.3390 /ijms 19020503

Leso V, Fontana L, Marinaccio A, Leopold K, Fanali C, Lucchetti D, Sgambato A, Iavicoli I (2018) Palladium nanoparticle effects on endocrine reproductive system of female rats. Hum Exp Toxicol 37:1069-1079

Leso V, Fontana L, Marinaccio A, Leopold K, Fanali C, Lucchetti D, Sgambato A, Iavicoli I (2019) Sub-chronic palladium nanoparticle effects on the endocrine reproductive system of female Wistar rats: preliminary data. Toxicol Ind Health 35:403-409

Liz R, Simard JC, Leonardi LB, Girard D (2015) Silver nanoparticles rapidly induce atypical human neutrophil cell death by a process involving inflammatory caspases and reactive oxygen species and induce neutrophil extracellular traps release upon cell adhesion. Int Immunopharmacol 28:616-625

Lu S, Duffin R, Poland C, Daly P, Murphy F, Drost E, Macnee W, Stone V, Donaldson K (2009) Efficacy of simple short-term in vitro assays for predicting the potential of metal oxide nanoparticles to cause pulmonary inflammation. Environ Health Perspect 117:241-247

Moeller S, Kegler R, Sternberg K, Mundkowski RG (2012) Influence of sirolimus-loaded nanoparticles on physiological functions of native human polymorphonuclear neutrophils. Nanomedicine 8:1293-1300

Murphy-Marion M, Girard D (2018) Titanium dioxide nanoparticles induce human eosinophil adhesion onto endothelial EA.hy926 cells via activation of phosphoinositide 3-kinase/ Akt cell signalling pathway. Immunobiology 223(2):162170. https://doi.org/10.1016/j.imbio.2017.10.030

Neubauer N, Palomaeki J, Karisola P, Alenius H, Kasper G (2015) Size-dependent ROS production by palladium and nickel nanoparticles in cellular and acellular environments - an indication for the catalytic nature of their interactions. Nanotoxicology 9:1059-1066

Noel C, Simard JC, Girard D (2016) Gold nanoparticles induce apoptosis, endoplasmic reticulum stress events and cleavage 
of cytoskeletal proteins in human neutrophils. Toxicol in Vitro 31:12-22

Pelletier M, Girard D (2005) Interleukin-15 increases neutrophil adhesion onto human respiratory epithelial A549 cells and attracts neutrophils in vivo. Clin Exp Immunol 141:315-325

Pelletier M, Bouchard A, Girard D (2004) In vivo and in vitro roles of IL-21 in inflammation. J Immunol 173:7521-7530

Petrarca C, Clemente E, Di Giampaolo L, Mariani-Costantini R, Leopold K, Schindl R, Lotti LV, Mangifesta R, Sabbioni E, Niu Q, Bernardini G, Di Gioacchino M (2014) Palladium nanoparticles induce disturbances in cell cycle entry and progression of peripheral blood mononuclear cells: paramount role of ions. J Immunol Res 2014:295092

Phan TTV, Huynh TC, Manivasagan P, Mondal S, Oh J (2019) An up-to-date review on biomedical applications of palladium nanoparticles. Nanomaterials (Basel) 10(1):66. https://doi. org/10.3390/nano10010066

Poirier M, Simard JC, Antoine F, Girard D (2014) Interaction between silver nanoparticles of $20 \mathrm{~nm}$ (AgNP20) and human neutrophils: induction of apoptosis and inhibition of de novo protein synthesis by AgNP20 aggregates. J Appl Toxicol 34: 404-412

Poirier M, Simard JC, Girard D (2015) Silver nanoparticles of $70 \mathrm{~nm}$ and $20 \mathrm{~nm}$ affect differently the biology of human neutrophils. J Immunotoxicol 13(3):375-85. https://doi. org/10.3109/1547691X.2015.1106622

Ravindra K, Bencs L, Van Grieken R (2004) Platinum group elements in the environment and their health risk. Sci Total Environ 318:1-43

Reale M, Vianale G, Lotti LV, Mariani-Costantini R, Perconti S, Cristaudo A, Leopold K, Antonucci A, Di Giampaolo L, Iavicoli I, Di Gioacchino M, Boscolo P (2011) Effects of palladium nanoparticles on the cytokine release from peripheral blood mononuclear cells of palladium-sensitized women. J Occup Environ Med 53:1054-1060

Savoie A, Lavastre V, Pelletier M, Hajto T, Hostanska K, Girard D (2000) Activation of human neutrophils by the plant lectin Viscum album agglutinin-I: modulation of de novo protein synthesis and evidence that caspases are involved in induction of apoptosis. J Leukoc Biol 68:845

Schleimer RP, Sterbinsky SA, Kaiser J, Bickel CA, Klunk DA, Tomioka K, Newman W, Luscinskas FW, Gimbrone MA Jr,
McIntyre BW et al (1992) IL-4 induces adherence of human eosinophils and basophils but not neutrophils to endothelium. Association with expression of VCAM-1. J Immunol 148: 1086

Simard JC, Simon MM, Tessier PA, Girard D (2011) Damageassociated molecular pattern S100A9 increases bactericidal activity of human neutrophils by enhancing phagocytosis. J Immunol 186:3622-3631

Skoczen SL, Potter TM, Dobrovolskaia MA (2011) Method for analysis of nanoparticle effects on cellular chemotaxis. Methods Mol Biol 697:247

Theron AJ, Ramafi GJ, Feldman C, Grimmer H, Visser SS, Anderson R (2004) Effects of platinum and palladium ions on the production and reactivity of neutrophil-derived reactive oxygen species. Free Radic Biol Med 36:1408-1417

Turner N, Armitage M, Butler R, Ireland G (2004) An in vitro model to evaluate cell adhesion to metals used in implantation shows significant differences between palladium and gold or platinum. Cell Biol Int 28:541-547

Vandooren J, Berghmans N, Dillen C, Van Aelst I, Ronsse I, Israel LL, Rosenberger I, Kreuter J, Lellouche JP, Michaeli S, Locatelli E, Franchini MC, Aiertza MK, Sanchez-Abella L, Loinaz I, Edwards DR, Shenkman L, Opdenakker G (2013) Intradermal air pouch leukocytosis as an in vivo test for nanoparticles. Int J Nanomedicine 8:4745

Wilkinson KE, Palmberg L, Witasp E, Kupczyk M, Feliu N, Gerde P, Seisenbaeva GA, Fadeel B, Dahlen SE, Kessler VG (2011) Solution-engineered palladium nanoparticles: model for health effect studies of automotive particulate pollution. ACS Nano 5:5312-5324

Yuan X, Nie W, He Z, Yang J, Shao B, Ma X, Zhang X, Bi Z, Sun L, Liang X, Tie Y, Liu Y, Mo F, Xie D, Wei Y, Wei X (2020) Carbon black nanoparticles induce cell necrosis through lysosomal membrane permeabilization and cause subsequent inflammatory response. Theranostics 10:4589-4605

Publisher's note Springer Nature remains neutral with regard to jurisdictional claims in published maps and institutional affiliations. 